\title{
Landsat Data Continuity Mission
}

\section{What Is the LDCM and Why Is It Important?}

The Landsat Data Continuity Mission (LDCM) is a partnership formed between the National Aeronautics and Space Administration (NASA) and the U.S. Geological Survey (USGS) to place the next Landsat satellite in orbit no earlier than February 11, 2013. The Landsat era that began in 1972 will become a nearly 41-year global land record with the successful launch and operation of the LDCM. The LDCM will continue the acquisition, archiving, and distribution of multispectral imagery affording global, synoptic, and repetitive coverage of the Earth's land surfaces at a scale where natural and human-induced changes can be detected, differentiated, characterized, and monitored over time.

The mission objectives of the LDCM are to (1) collect and archive medium resolution (30-meter spatial resolution) multispectral image data affording seasonal coverage of the global landmasses for a period of no less than 5 years; (2) ensure that LDCM data are sufficiently consistent with data from the earlier Landsat missions in terms of acquisition geometry, calibration, coverage characteristics, spectral characteristics, output product quality, and data availability to permit studies of landcover and land-use change over time; and (3) distribute LDCM data products to the general public on a nondiscriminatory basis at no cost to the user.

\section{LDCM History}

The original LDCM plans called for NASA to purchase data meeting LDCM specifications from a commercially owned and operated satellite system; however, after an evaluation of proposals received from industry, NASA cancelled the Request for Proposals in September 2003. In August 2004, a memorandum from the White House Office of Science and Technology Policy (OSTP) directed Federal agencies to place Landsat-type sensors on the National Polar-orbiting Operational Environmental Satellite System platform. Following an evaluation of the technical complexity of this task, the strategy was adjusted and on December 23, 2005, the OSTP issued a memorandum directing NASA to implement the LDCM in the form of a free-flyer spacecraft carrying an instrument referred to as the Operational Land Imager (OLI). NASA and the USGS are now implementing the OSTP directive. In December 2009, a decision was made to add a thermal infrared sensor (TIRS) to the mission payload.

\section{Implementing the LDCM}

The LDCM is being developed with a 5-year mission design life, yet will include enough fuel for 10 years of operation. NASA and the USGS share responsibility for LDCM implementation. NASA will develop the flight systems, including the spacecraft, the instrumentation,
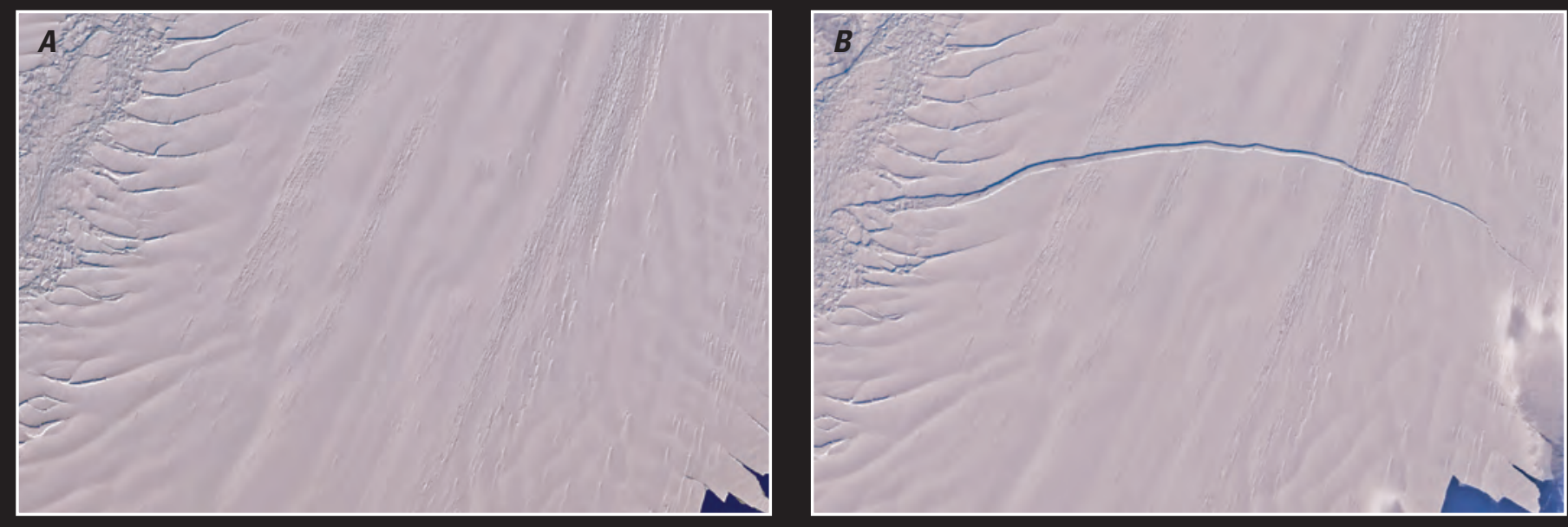

The breaking of Pine Island Glacier in Antarctica. A, Image acquired January 25, 2011. B, Image acquired January 28, 2012. 
the mission operations element, the mission launch, and perform the on-orbit checkout. The USGS will develop, implement, and operate the ground network for data acquisition, data archive, processing facilities, and distribution of data products. In addition, the USGS will be responsible for satellite flight operations following launch.

The centerpiece of the LDCM space segment is the OLI. By collecting land-surface data with spatial resolution and spectral band specifications consistent with historical Landsat data, the OLI instrument will advance future measurement capabilities while ensuring compatibility with historical data. The OLI will feature two additional spectral channels: a deep-blue band for coastal water and aerosol studies, and a band for cirrus cloud detection. The TIRS will collect data in two long wavelength thermal bands that will be co-registered with OLI data (table 1).

Table 1. Planned parameters for Landsat Data Continuity Mission (LDCM) standard products.

\begin{tabular}{ll}
\hline Product type & Level 1T (terrain corrected) \\
Output format & GeoTIFF \\
Pixel size & $\begin{array}{l}15 \text { meters/30 meters/100 meters } \\
\text { (panchromatic/multispectral/thermal) }\end{array}$ \\
Map projection & UTM (Polar Stereographic for Antarctica) \\
Datum & WGS 84 \\
Orientation & North-up (map) \\
Resampling & Cubic convolution \\
& \\
Accuracy & OLI: 12 meters circular error, 90-percent confidence \\
\end{tabular}

A key feature in the ground segment being planned by the USGS is the provision of high-quality LDCM standard data products. About 400 scenes per day will be imaged over global land and coastal areas and returned to the United States for archive, processing, and distribution. All acceptable scenes will be terrain corrected to a geographic projection and made available at no cost to users by way of the Internet.

\section{Landsat Science Team}

An important component of the LDCM is the Landsat Science Team, funded by the USGS and led by the USGS and NASA. The team works to advance the objectives of LDCM and to contribute to the integration of LDCM data with past, present, and future Landsat and other remotely sensed data. The team also provides science support on issues critical to the success of the mission, including data acquisition, product access and format, and science 

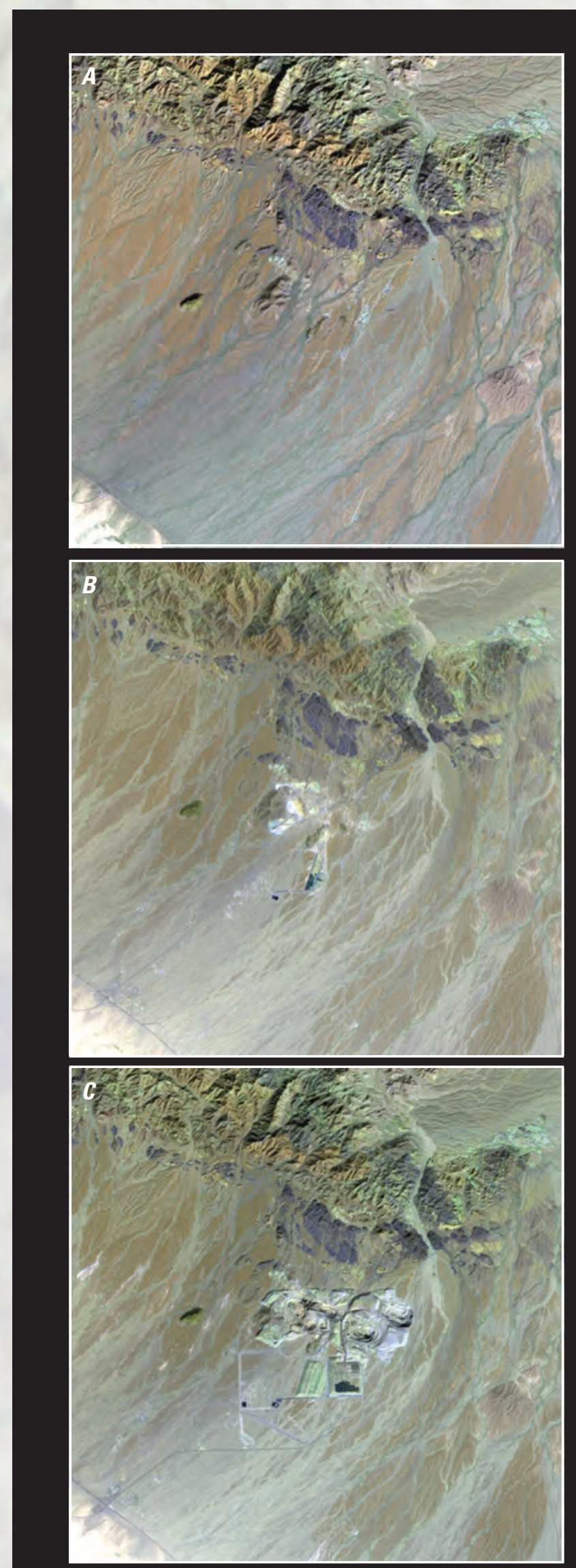

The growth of the Mesquite Mine in California. $A$, Image acquired December 12, 1982. $B$, Image acquired July 27, 1987. C, Image acquired November 2, 2011. opportunities. The members listed below served from 2006 to 2011. Another team will be selected through a competitive solicitation in 2012.

- Richard Allen, University of Idaho

- Martha Anderson, U.S. Department of Agriculture, Agricultural Research Service

- Alan Belward, Joint Research Center of the European Commission

- Robert Bindschadler, NASA Goddard Space Flight Center

- Warren Cohen, U.S. Department of Agriculture, Forest Service, Pacific Northwest Research Station

- Feng Gao, Earth Resources Technology

- Sam Goward, University of Maryland

- Dennis Helder, South Dakota State University

- Eileen Helmer, U.S. Department of Agriculture, Forest Service, International Institute of Tropical Forestry

- Rama Nemani, NASA Ames Research Center

- Lazaros Oreopoulos, University of MarylandBaltimore County

- John Schott, Rochester Institute of Technology

- Prasad Thenkabail, U.S. Geological Survey

- Eric Vermote, University of Maryland

- James Vogelmann, U.S. Geological Survey

- Curtis Woodcock, Boston University

- Michael Wulder, Canadian Forest Service

- Randolph Wynne, Virginia Polytechnic Institute

\section{Looking Forward}

The U.S. Government seeks to ensure continuity of Landsat-like data. As stated in the December 2005 OSTP memorandum: "Concurrent with the actions cited, the National Science and Technology Council, in coordination with NASA, DOI/USGS, the Executive Office of the President, and other agencies and offices as appropriate, will lead an effort to develop a long-term plan to achieve technical, financial, and managerial stability for operational land imaging in accord with the goals and objectives of the U.S. Integrated Earth Observation System." Toward this end, the OSTP released a plan in August 2007 for a National Land Imaging Program that includes calling for operational moderate resolution land imaging. The National Space Policy of the United States, issued June 28, 2010, states, "The Secretary of the Interior, through the Director of the USGS, and the NASA Administrator shall work together in maintaining a program for operational land remote sensing observations." 


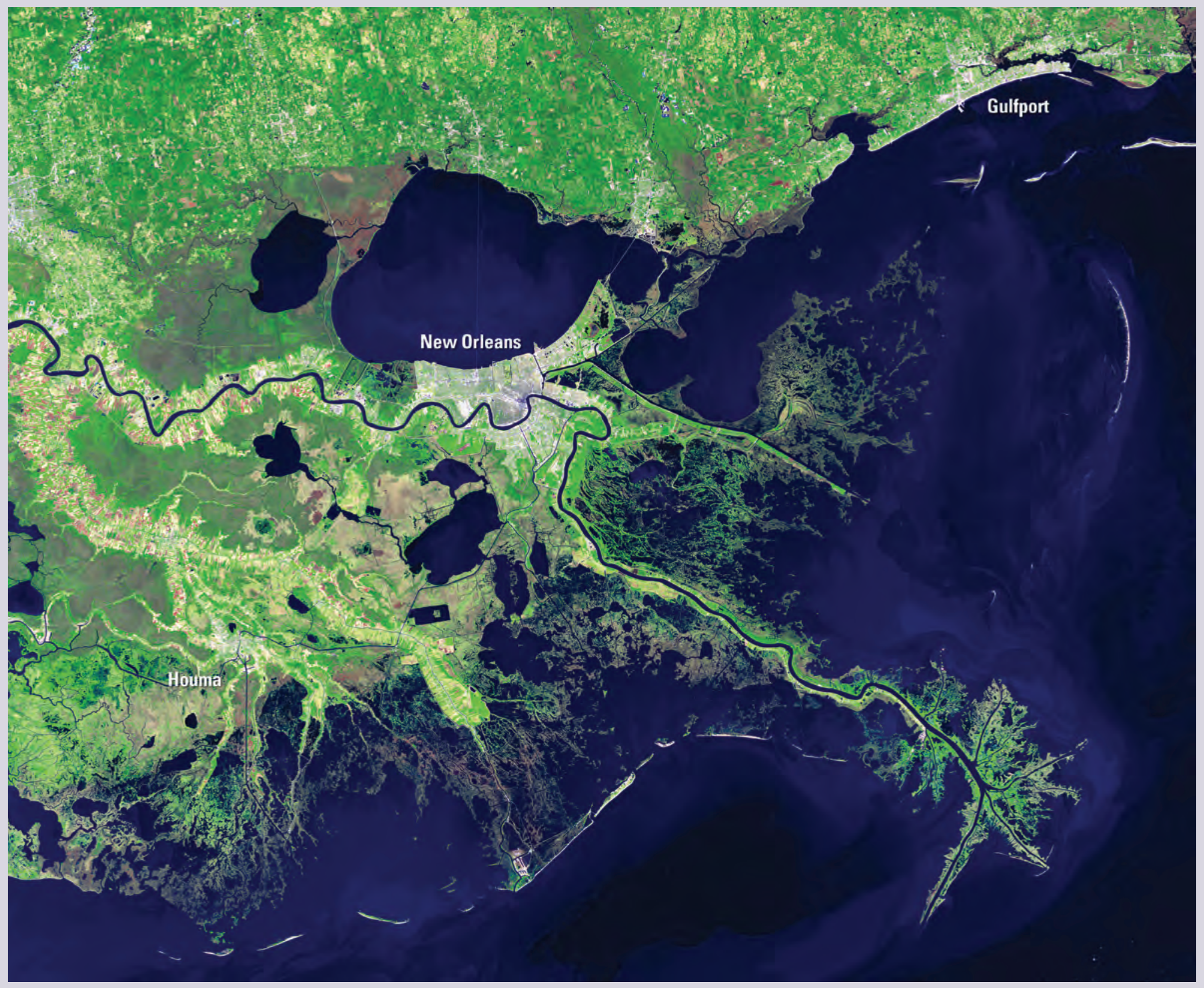

The Mississippi River Delta Basin. This image was created by mosaicking four Landsat scenes acquired October 3 and November 11, 2011.

For information on LDCM and to receive updates and newsletters, visit http://ldcm.usgs.gov, http://ldcm.nasa.gov/,

or contact:

U.S. Geological Survey

Earth Resources Observation and Science (EROS) Center

Mundt Federal Building

Sioux Falls, SD 57198

Phone: 605-594-6151

Toll free: 800-252-4547

Fax: 605-594-6589

Email: landsat@usgs.gov 